\title{
Co-located Collaborative Visual Analytics Around a Tabletop Display
}

\author{
Petra Isenberg Danyel Fisher Sharoda A. Paul Meredith Ringel Morris Kori Inkpen Mary Czerwinski
}

\begin{abstract}
Co-located collaboration can be extremely valuable during complex visual analytics tasks. We present an exploratory study of a system designed to support collaborative visual analysis tasks on a digital tabletop display. Fifteen participant pairs employed Cambiera, a visual analytics system, to solve a problem involving 240 digital documents. Our analysis, supported by observations, system logs, questionnaires, and interview data, explores how pairs approached the problem around the table. We contribute a unique, rich understanding of how users worked together around the table and identify eight types of collaboration styles that can be used to identify how closely people work together while problem solving. We show how the closeness of teams' collaboration and communication influenced how they performed on the task overall. We further discuss the role of the tabletop for visual analytics tasks and derive design implications for future co-located collaborative tabletop problem solving systems.
\end{abstract}

Index Terms-Co-located collaboration, tabletop displays, collaborative information visualization, complex problem-solving

\section{INTRODUCTION}

$\mathrm{V}$

ISUAL analytics tasks can have amorphous structure, ambiguous goals, and large amounts of data [1]. Many visual analytics problems, therefore, can be best solved by groups of analysts working together, face-toface. For example, Chin et al. [2] found that intelligence analysts highly value the ability to collaborate face-to-face: "It's when all of our analysts get together and work out the differences and challenge each other with facts that we get to a better and more prominent answer." Despite these benefits, we have surprisingly few technologies to support co-located visual analytics: instead, teams typically share one screen or work on separate computers, side by side. New technologies, such as interactive surfaces, offer increased screen real estate and multi-touch capabilities to build novel face-to-face collaborative workspaces. Yet, there have been few studies reporting on design and use of collaborative visual analytics workspaces for interactive surfaces. We try to fill this gap by providing a rich description of pairs working collaboratively, face-to-face, on a visual analytics task, using a tabletop system designed specifically for these tasks. We studied teams of two people solving the VAST 2006 Challenge, an intelligence analysis task that involves the exploration of a large text document collection. This task resembles many other scenarios in which pairs collaboratively attempt to solve complex problems over a large text corpus: for instance, lawyers making sense of a set of case files, military intelligence analysts trying to comb through millions of documents, coauthors researching old newspapers for help with a new story, or historians browsing through historic texts to bring past events into context. In these and many other tasks, analysts have considerable experience working face-to-face on traditional physical tables [2]. Our goal is to more fully understand the consequences of providing a digital tabletop analytics environment that incorporates the benefits of a shared space for synchronous collaborative work as well as computational support for searching and data sharing. Studying and formalizing the nature of collaborative analysis activities within such an environment is valuable to inform our understanding of digital tabletop visual analytics systems and provides valuable design advice for future systems, as we shall discuss. We begin by reviewing the Cambiera system [3], and then present a study and discuss our observations. This article extends a previous conference paper [4] and adds new detail on how participants used the workspace during the study and their activities off and around the tabletop display.

Our goal is to address the digital table as a context for colocated visual analytics: to describe how pairs approached the problem on the table effectively; to discuss what features helped pairs with the analysis task, and to describe what additional features would be even more beneficial in supporting complex problem solving on the tabletop.

\section{Related Work}

Our study is a rich examination of how teams communicate and coordinate face-to-face around a visual analytics tabletop system. While researchers have explored various tools for visual analytics support, our focus on co-located tabletop collaboration is unique and revealed novel patterns of interacting that have not before been reported thoroughly. In this section, we discuss work that has inspired our own through a focus on collaborative work with complex analysis tasks and visualization support.

\subsection{Distributed Collaborative Problem Solving}

Most research projects exploring collaborative problemsolving have supported distributed, rather than face-to- 


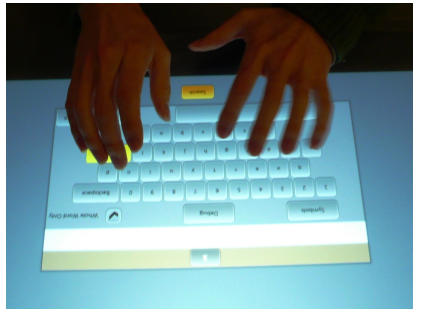

(a) Analysts begin searching for data.

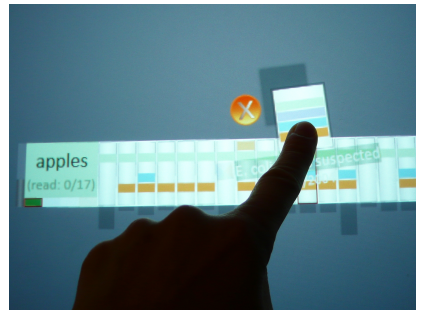

by (b) A document is pulled out of a search result list.

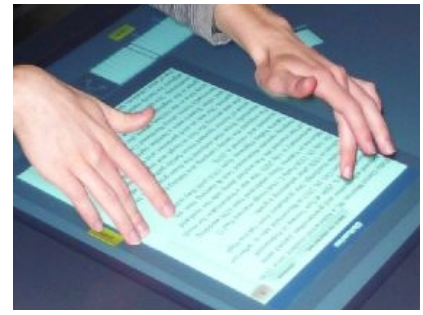

(c) A document is zoomed to be read and analyzed.

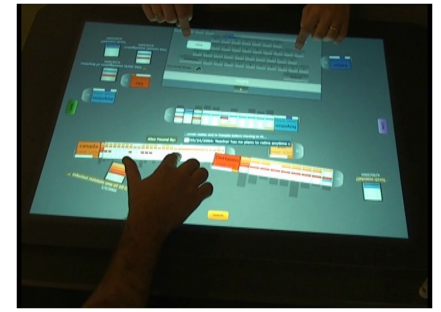

(d) Items can be freely organized and shared.

Fig. 1. Several different aspects of the Cambiera system in use during a document analysis task.

face, collaboration. For example, Balakrishnan et al. [5] addressed the question of how teams can share visualizations remotely to solve a complex problem. They found that a visualization was most effective when both partners had full access to the shared visualization and could both synchronously interact with it; in a follow-up study [6], they further found that collaborators were more effective when they each had control over parts of the data. We include an implicitly shared context in our system, where we give each analyst independent access to all data items and data interactions on the table. Brennan et al. [7] take a slightly different approach by requiring explicit sharing and merging of data views during distributed analysis. This way, team members can work alone on a subtopic, and then switch to a shared view that supports joint sensemaking. Like our system, Paul and Morris' search-oriented CoSense [8] provides collaborators with awareness support: teammates can see each other's web search histories, as well as the documents that they each have found, in order to support collaborative information seeking. Keel's system [9] for distributed analysts provided awareness by analyzing information from a team member's workspace and suggesting relevant data to other remote analysts, allowing for implicit information sharing. In contrast to these systems, ManyEyes' [10] social visualizations connect people who may not have any previous relationship. People can asynchronously share data, create visualizations, and collaboratively explore, annotate, and comment on the visualizations. In contrast to these systems for distributed problem solving, our work addresses face-to-face collaboration around a shared display and fundamentally differs in terms of the workspace setup and the ability for team members to communicate quickly and effortlessly about the data on the surface. In addition, it allows team members to distinguish themselves in meaningful and useful ways. We included some of the features employed earlier for distributed systems-such as support for parallel work and joint search histories-in our system and study, as they seem to be valuable.

\subsection{Co-located Collaborative Problem Solving}

Tabletop technologies can allow teams to easily share tools and information face-to-face, and researchers are still learning how best to leverage tables for complex tasks. Research on collaborative visual analytics on tabletop displays is still in its infancy (e.g., [11]). We therefore discuss work from the Tabletop Community that addresses more complex analysis tasks.

WeSearch [12] is related to our work in that it supports search, browsing, and sensemaking activities. Team members can share web searches, snippets, and annotations during their investigations. The DTLens system [13] demonstrates how focus+context techniques can be used for exploring large maps and diagrams on an interactive tabletop. DTLens addresses an important issue for collaborative problem solving: when multiple people simultaneously interact with information, parallel exploration may be hindered by team members' different interests. DTLens resolves this with a notion of ownership, in which individuals can only edit their own views of the data. In contrast, CoTree, a collaborative tabletop system for the comparison and analysis of hierarchical data [14] allows collaborators to use multiple independent views and visualizations of the same dataset. These can be explored in parallel or brought together for comparison without any sense of ownership. WeSpace [15] addresses the issue of providing individual views, and parallel exploration by allowing researchers to bring their own visualization applications on their own laptops to a multi-display environment. The table here is used to coordinate views from the different laptops on a wall around which shared viewing, discussion, and interpretation can happen. However, as the display is controlled by individual laptops, the system does not specifically facilitate simultaneous interaction with the shared views of the data. In our system we combined several of these approaches. We allowed collaborators to share data and work with multiple views of the same data source. We also made ownership explicit through color-coding but did not restrict view access. The next section describes these features in more detail. Finally, while a number of systems have been previously developed to support the complex tasks involved in intelligence work (e.g., [7], [9], [16]), to our knowledge there have been no studies of other systems in use in a tabletop context supporting visual analytics tasks.

\section{Cambiera: System Description}

Our study examines pairs analyzing a shared document collection using Cambiera [3]. Cambiera is one of the 


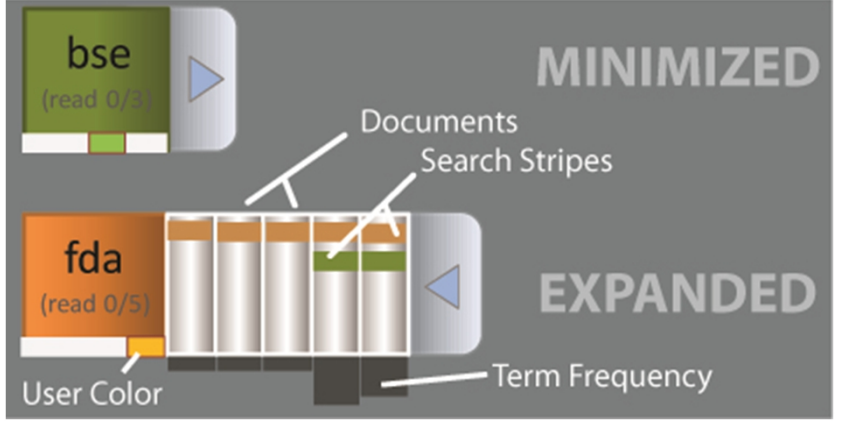

Fig. 2. Two search widgets. The top search widget ("bse" is minimized. The bottom widget is maximized; each vertical bar represents a document that contains the word "fda." The documents are ordered by publication date. The fourth and fifth documents also have green stripes and contain the word "bse."

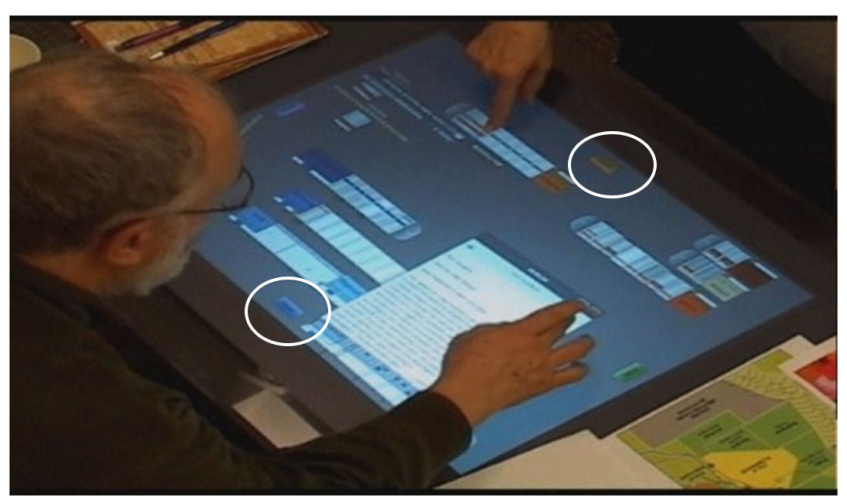

Fig. 3. Overview of the system in use. Each person issues searches starting from their own search button in front of them (highlighted by circles). Touching the button brings up a keyboard (Figure 1(a)) to type search terms and issue the search. Here the left analyst issues blue, and the right issues orange searches.

first tabletop systems designed specifically for co-located collaborative visual analytics. It currently runs on the Microsoft Surface, a multi-touch tabletop display. As a tabletop system, Cambiera encourages analysts to face each other around the tabletop, while analyzing large text document collections collaboratively. Cambiera features four fundamental operations:

Search for documents: Each team member starts their analysis with Cambiera by searching for relevant documents. Pressing one of the search buttons along the sides of the table calls an on-screen keyboard for entering a search term (Figure 1(a) and Figure 3). Each keyboard specific to and oriented towards just one analyst returns a color-coded search result widget, which holds virtual representations of the documents returned (Figure 2). Frequency bars under each document represent the total number of times the respective search term occurs in the document. The color hue of the widget is determined by the team member who issued the search. While hues between search words are only subtly different (and each analyst may not remember them in detail), each person is vividly distinct; in addition, the analyst can touch a document to see what words are associated with it.

Search result exploration: Each search widget can be expanded to see the returned list of documents ordered by their publication date (Figure 2). By running a finger across the document representations, detail-on-demand information is shown in an off-set information box next to the finger [3]. This information includes who has previously issued the search, what other search terms (regardless of the searcher) found the same document, and which sentences contained the search term.

Document analysis: documents can be pulled out of search result lists (Figure 1(b)) and then freely placed. By performing a zooming gesture the document's text can be accessed within a document reader (Figure 1(c)).

Workspace organization: All objects in Cambiera can be moved around the table, re-oriented, resized, or closed. Each analyst can place and stack documents and search results on the tabletop as they find most useful (Figure 1(d)). An analyst can also spin the documents around in order to push them to the other side, one of the several ways that Cambiera allows people to share documents.

One of the unique features of Cambiera is its support for co-located awareness. Each individual search widget is tagged with a unique, user-dependent color. Each document within the search widget in turn is annotated with colored stripes corresponding to the search terms that have been found within them. Because each search widget has its own color, the colored stripes on the search results indicate when they have been found by more than one word. In Figure 2, for example, one team member has searched for "FDA" (Federal Department of Agriculture); another has searched for "BSE" (Bovine Spongiform Encephalitis). The colored stripes indicate that the last two documents found by the search for "fda" also contain the word "bse." When documents are pulled out of a search result list, they maintain the colored bars that indicate which search generated them. In the document reader (Figure 1(c)), the text highlights all search terms that have found this document, regardless of which team member issued them. In addition, all states of a document include a specific icon (in the search result list, minimized in the workspace, and maximized in the reader) which indicates whether the document has been read, by whom and the shading of the document's background color gives a relative indication of read frequency. Since previous studies of collaborative data analysis have shown that team members frequently switch to phases of parallel work [17], [18], these awareness features were designed to encourage co-located team members to connect more closely with others and to be able to more easily synthesize their individual analysis results.

\section{USER STUdy}

To design visual analytics tabletop tools for co-located collaborative work we need to understand how these systems are used by teams. In particular, we need a better 
understanding of how teams coordinate their activities over a tabletop and which specific system features are best suited to support effective co-located collaborative data analysis in this context. Our study was designed to provide a rich description of collaborative visual analysis on a tabletop display targeting this information need. In this exploration, we were guided by questions of understanding collaborative analytics on the tabletop.

- How do teams work together and coordinate their work with the tabletop?

- What patterns of collaboration do they carry out?

- How can their work patterns be used to inform the design of future interfaces for collaborative problem solving?

\subsection{Participants}

It is extremely difficult to obtain professional intelligence analysts for real-life intelligence analysis scenarios; therefore, we followed the procedures used by the VAST 2006 Challenge [19], which provides a baseline task that closely models an analysis scenario, but can be carried out by nonanalysts. We describe the task further below. We recruited 15 pairs of people who were familiar with data analysis. Participants were required to have a Master's (or more advanced) degree, and to have self-reported as enjoying puzzles or mysteries. The members of each pair knew each other and had previously worked together in some form; subjects were co-workers, friends, family members, and married couples. Participants ranged in age from 2555 ; ten couples were mixed-gender; three both women; two both male. On average participants spent 72 minutes $(\operatorname{stddev}(\sigma)=12)$ working on the task.

\subsection{The VAST 2006 Challenge}

We based our experiment around the VAST 2006 Challenge, code-named "Stegosaurus" [19], a scenario that entails finding a hidden weapons-smuggling plot. Stegosaurus was developed by the National Visual Analytics Center at Pacific National Labs. We chose "Stegosaurus" because it is increasingly recognized as a standard visual analytics task [20]. The dataset is available to the public to download, and is usable by non-experts without training. Nonetheless, it is a complex task that requires participants to connect and infer facts buried in a set of several hundred documents. We used an extended version of the Stegosaurus document set containing 240 digital and 6 paper documents. Cambiera does not currently support search of non-textual materials like maps and images. Thus, we preloaded Cambiera with all of the newspaper and fact sheet articles but provided paper print-outs of the map and other images.

The task comes with a vague goal of figuring out a car crash; from there, the analyst needs to work through the dataset. No single document tells the whole story-of the 246 documents, just ten have true bearing on the story, while several others provide some background information but are not necessary, and the rest are irrelevant. Solving the task requires carefully filtering out irrelevant articles, reading articles in detail, and making several intuitive leaps. In between stories about fruit-picking season, one document tells us that people who ate at an apple festival got intestinal poisoning, probably from tainted meat. A second article says that a batch of apples stored in a silo was contaminated with nerve gas. It requires searching, reading, and intuition to recognize that the symptoms of nerve gas were likely mistaken for intestinal poisoning, and that they came from the same apples. Collaboration has the potential to improve the effectiveness of the analysis in several ways. Teams can cover more documents in the same amount of time, can discuss evolving hypotheses and contribute facts they may have found individually. At the same time, collaboration can be a mixed blessing if team members fail to share and/or synthesize their results. The goal of our evaluation is to study more closely how teams work together in solving this task with our tool, and to derive further requirements and guidelines for tabletop visual analytics tools.

\subsection{Procedure}

Our study used the VAST 2006 challenge data loaded in Cambiera. Participants sat on opposite sides of the table and received a fifteen-minute tutorial on the features of Cambiera using a sample dataset, during which they were encouraged to experiment with the system and ask questions freely. They were read an introductory letter to the "Stegosaurus" problem, which explained the task context. That letter includes a starting clue that suggested a first document to read. Since our focus was on observing the pairs' collaborative interactions with each other and with the system, and less on performance outcomes, we wanted to ensure that they were able to make progress in the task and continued working. For this reason, we provided assistance to teams who did not progress in the task. The experimenter running the study was familiar with the dataset, and so was able to monitor teams' progress. When teams stopped making progress, as judged by the experimenter (e.g., reading and re-reading distracter documents or reporting to be stuck) the experimenter provided assistance. During an assist, the experimenter did not provide new information, but rather asked the participants to clarify previous ideas that they had raised. We maintained consistency of assists by using only one experimenter, who followed a written protocol. Participants were also provided with pens and notepads for taking notes, and a small set of auxiliary images that are part of the "Stegosaurus" scenario. Participants reported their results verbally at the end of the study. We terminated the experiment when the team could produce a coherent story when asked for their hypotheses, and ended all experiments at one and a half hours. After the study, participants independently filled out a questionnaire (due to a technical error, three pairs did not fill out the questionnaire). The questionnaire elicited information on participant demographics and subjective awareness experience. Finally, the experimenter interviewed the pair to understand how they approached the problem and to get their feedback on 
the technology. Originally, we designed three experimental conditions that varied aspects of Cambiera's visualization; in the different conditions, three different color schemes were used to subtly convey information about which documents one's partner had read and which terms they had searched for. In a formal statistical analysis of the data we did not find significant differences between the number of searches performed, documents read, documents/searches passed between participants, or facts connected in each condition. Thus, we report observations that apply to all pairs, regardless of condition.

\subsection{Data Analysis}

In order to gain a rich understanding of how the tasks were solved we captured a variety of sources: each experimental session was video- and audio-recorded; in addition, the software captured a screenshot every minute and a timestamped event $\log$ for interactions with the tabletop. One experimenter took notes in real time; a second experimenter engaged in a two-pass video coding. During the first video coding pass the following information was coded: collaboration styles based on a code set by Tang et al. [18], roles adopted by participants, use of awareness features, use of external information (shared or private notes), breakdowns or conflicts in group work, and how often workspace items were shared. After the first coding pass it became evident that the code set from Tang et al. [18] had to be extended to accommodate the different study situation. Whereas the original code set was developed for a situation in which participants shared the same representation, our participants could work with data, representations, and views of the data in parallel. Therefore, we extended the code set to more clearly distinguish when people shared views of the same data and when they were looking at the same data but using copies of the same information items and thus had different views of the same information. Details on the extended codes follow in Section 5.3. During the second coding pass, the video coder also took more detailed time-stamped notes on when participants switched to different types of collaboration styles using the extended code set as well as extended notes on which facts and documents teams found as they progressed through the task. A second video coder analyzed three representative videos to understand the verbal communication among team members. This coder transcribed all verbal communication and combined the transcriptions with the log files to gain a comprehensive understanding of the actions performed by each pair. The transcripts were coded in several passes, first using an open coding technique looking for themes related to sensemaking. In a second pass, the transcripts were coded using a coding scheme derived from Pirolli and Card [21]. Finally, written transcripts of participants' answers to the post-session questionnaire were coded into higher-level categories including awareness (e.g., which information was missing or helpful), work styles (strategies, roles, sharing, and collaboration), and tool features commented on (liked and missing features). The log data was further parsed and analyzed using descriptive statistics to derive information on the frequency of use of system features such as the keyboard, document readers, or the search result lists. The most important numbers are listed in the results sections. We also wrote Pairgams, a visualization tool for graphical overviews of participants' individual and collaborative search behavior. We used Pairgrams in conjunction with video analysis (see [22] for details).

Notes taken by participants and used during the experiment were also collected and analyzed. Together, the detailed analysis of video, field notes, system logs, communication patterns, and interview data provided a rich understanding of how participants solved the task, worked with Cambiera, and how they engaged with each other.

\section{Solving a Complex Problem Col- LABORATIVELY}

With the exception of some research papers [20], [2] and the VAST contest reports, there are few descriptions of how users work on complex analysis tasks, and none of them discuss collaborative aspects in detail [23]. In this section, we describe the major strategies our participants adopted to solve the complex VAST challenge.

\subsection{Starting from a First Clue}

The introductory letter read to the participants explicitly mentioned an initial document; all participants started by finding this initial document. Next, teams chose different strategies to collaborate or divide their work: in nine of the teams, both partners worked together to read the first article: either one member would wait while the other found the article, or both searched for it in parallel. In the other six teams, one team member volunteered to read the document; the other began to search for other terms they believed to be relevant ("conspiracy"), or searched for interesting locations on the provided map ("missile silo sounds interesting").

\subsection{Iterative Searching and Reading}

After working on the first document, teams engaged in an iterative process of searching for and reading documents they had found. The task required participants to connect facts from a number of different documents; this is reflected in the large number of documents that participants searched for, found, and read. Teams issued on average 50 searches with 42 unique search terms. An average of 90 of the 240 documents were then selected from these searches. $70 \%$ of these documents were opened and read by one or both team members (63 documents on average). Interestingly, 58 documents on average were read more than once. We observed that participants revisited previous documents after they or their collaborator had an insight about their work so far, or found new information in other documents. The remaining $30 \%$ of all documents pulled out of the list were never opened but left on the tabletop. We suspect that these documents were kept to be checked later and finally determined to be unnecessary. 


\subsection{Information Sharing and Collaboration}

As participants worked on the dataset, they adopted different collaboration styles. At times, they would work on the same problem, even reading a single document together; at other times, they would separate to work on different problems. These different collaboration styles allowed them to flexibly investigate temporary hypotheses, test ideas, and continue to build a narrative. In order to more closely understand which information and views of the data space participants were sharing and collaborating on at a given moment, we coded their interactions based on their data views and personal interactions. We identified the following eight different collaboration styles and activities that participants adopted (see below for illustrations). These extend previous work by Tang et al.; underlined abbreviations are styles not found in the original publication [18]:

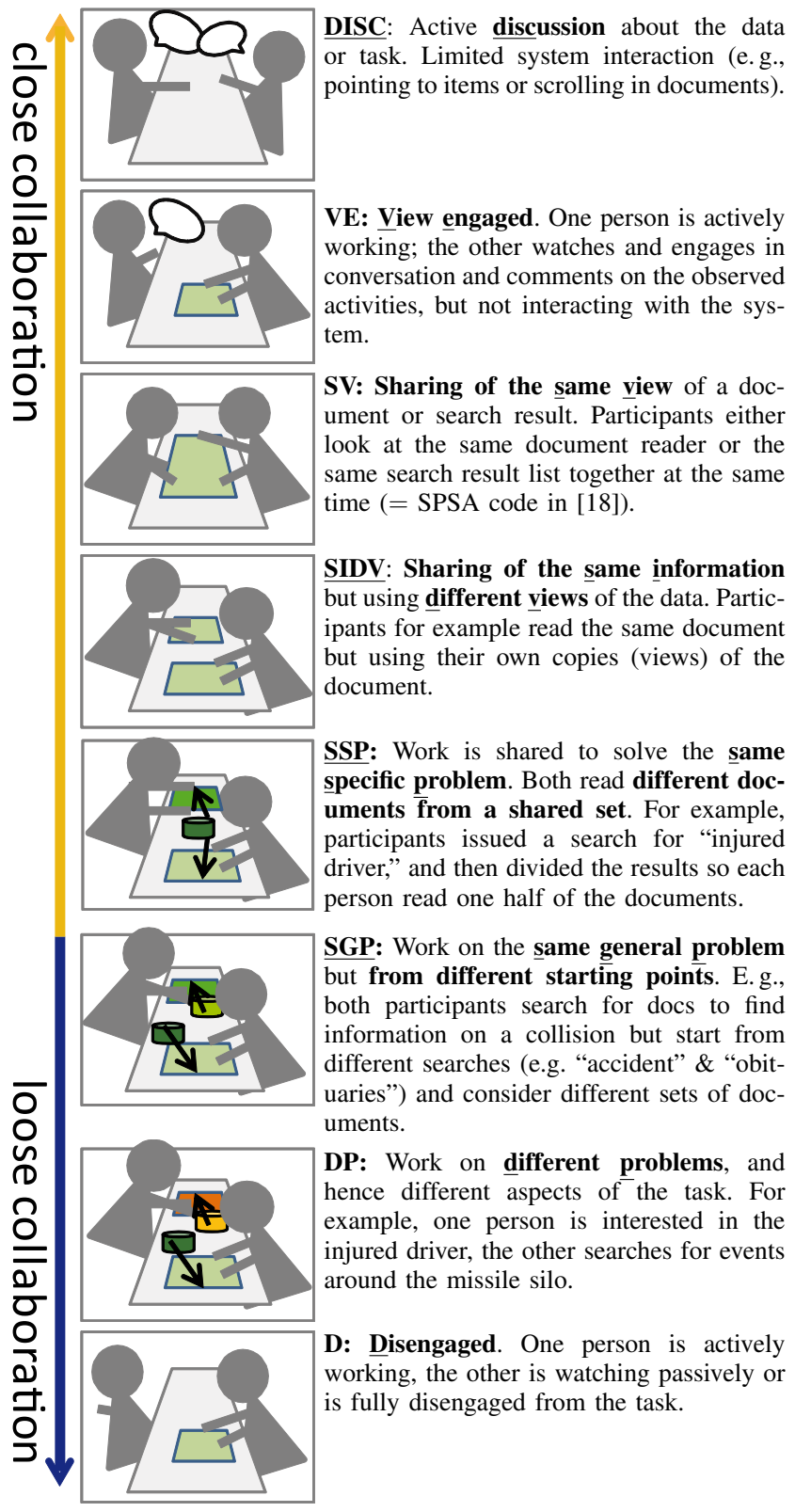

During the video coding we recorded time-stamped event-logs for each of these collaboration categories. We further grouped them into those describing 'close' collaboration (the first five codes) and 'loose' collaboration (the last three codes). Close collaboration was generally characterized by active sharing of information and discussion of hypotheses. During phases of close collaboration, teams shared these temporary hypotheses, looked at related information, and pursued similar questions. During phases of loose collaboration, explicit verbal sharing of information and evolving hypotheses was less frequent as team members were looking at less related information. Our coding revealed that teams showed high task engagement, with almost no time spent disengaged (D) from the task ( $<1 \%$ of the time on average). We observed a tendency for groups to share information and hypotheses frequently, with eleven (of 15) groups spending over half of their time in close collaboration. We refer to those eleven groups as closely coupled; the remaining four are loosely coupled.

In loosely coupled teams, participants spent on average $60 \%$ of their time working in parallel, and only infrequently interacted with their team members. Loosely coupled teams spent the dominant portion of their time pursuing different searches and ideas, but working on the same general problem (SGP). In SGP, teams were trying to answer a common general question such as "what is the involvement of Boynton laboratories" but started from different searches. The video coding revealed that $43 \%$ of their total task time was spent in this condition. In these groups, team members worked largely in their own part of the workspace with separate search results and documents; they would discuss (DISC) what they had found $7 \%$ of the time. The bottom four groups in Table 1 were loosely coupled groups.

Closely coupled groups spent on average $70 \%$ of their time closely interacting with the other team members. They spent the most time working from a shared set of documents (SIDV, taking $24 \%$ of their total task time). They were also likely to examine the same views as each other (SV, 13\%). Further evidence for close interaction between participants in closely coupled groups is the overall discussion time (DISC) with $11 \%$, and the time spent discussing and analyzing the same views (SV) of the data with $13 \%$ of the total task time, compared to just $5 \%$ for loosely coupled teams. Table 1 gives an overview of how much all groups spent in either closely or loosely couples phases. How much information participants shared and how frequently they connected to others had an influence on how well teams were able to connect the facts and progress through the task. We discuss this in more detail in Section 8.

\subsection{Presenting the Solution}

Groups were successful in finding the documents required to solve the task. Eleven of the 15 groups found all ten critical documents; the remainder missed one or two. It is worth noting that while the dataset was large, all teams successfully found most of the critical documents in the first half the experiment. While participants had found most of the relevant information by that time, however, they had 
TABLE 1

Diagrams of coupling styles for each pair. The second column indicates \% of time spent closely coupled. Blue phases in the diagrams encode loosely coupled and yellow closely coupled phases. White indicates phases in which groups stopped working (e.g., for interaction with the experimenter).

\section{Pair \% C.C. Coupling Styles}

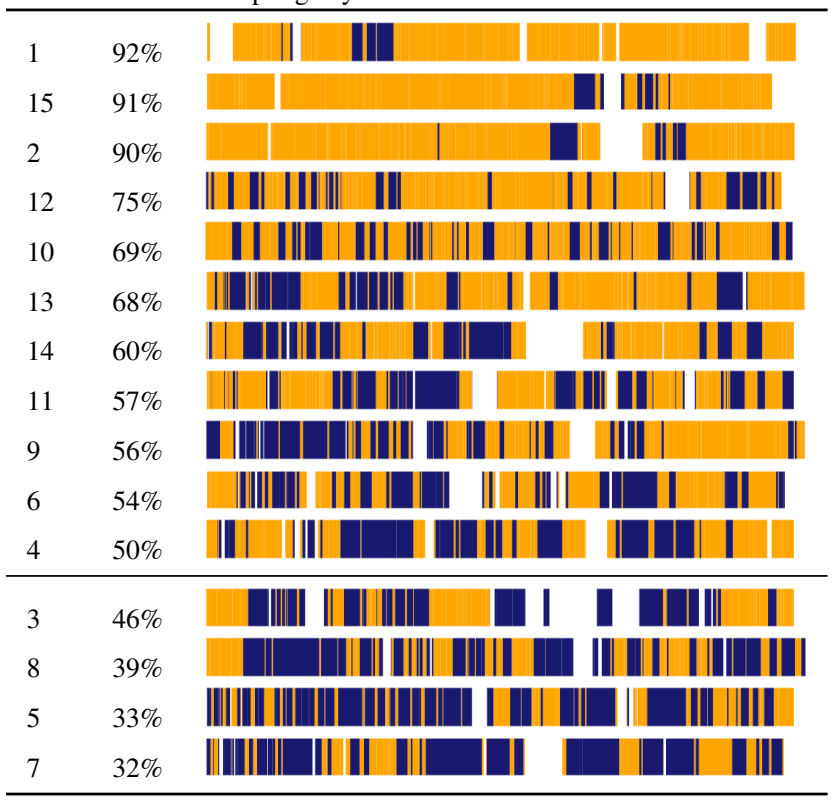

typically not managed to connect the pieces of information they had into a coherent hypothesis. During the study, the experimenter paused the teams half-way through to find out what they thought was going on. Much to our surprise, members of several of the teams had radically different working hypotheses from their partners: in one dramatic example, one participant had been pursuing a hypothesis centered around a political intrigue, while the other was working on a terrorism question. As a result, the checkpoint turned out to be accidentally invaluable for several of the teams. The checkpoint was sometimes the first time when participants would link subtle details together: one person would express a hypothesis ("it was e-coli poisoning") and the other would realize that an intuitive leap had been missed ("no, the apples were poisoned!"). After the checkpoint, however, teams began to better find the value of coordinating; almost all of the teams had a unified story by the last stage.

\section{Use of the Tabletop Workspace}

We further analyzed the activities on the tabletop workspace to understand how teams used documents and searches and how this reflected the thoughts and reasoning activities of individuals and teams as a whole. Given the large number of searches issued and documents pulled out of search results, organization of information items on the workspace was an important issue to handle.
We observed information organization behaviors common to several groups in the study. We found that people tended to adopt different organization strategies depending on their progress within the analysis task and depending on their main analysis activities. During phases in which team members read and searched for information independently they began to organize their work in their personal workspaces directly in front of them. These areas of the workspace have been previously studied as personal territories [24] in which people perform independent activities. We similarly observed independent reading, searching, and browsing activities in these spaces. The pairs tended to work more closely when discussing their findings, clarifying information, or forming hypotheses; it was in these more collaborative phases that the more centralized space was used for joint reading and validation. Once the team had made progress on the task, found some partial answers, and was ready to form hypotheses and connect the facts, then team members would switch to a shared document organization. Every pair adopted several different strategies throughout the task. Cambiera does not have any foldering, clustering, or grouping methods for objects; thus, we observed how pairs manually organized the space depending on their progress throughout the task. We identified three specific strategies that teams and individuals adopted:

Early Organization Strategy-Spread: At the beginning of the task many participants did not organize their documents carefully, instead letting them fall as they maymainly arranging documents so that they would not cover others (Figure 4). Eleven pairs adopted this strategy for at least part of the study. Documents and searches tended to remain oriented toward the person who had last used them, indicating ownership. We observed a tendency for pairs to spread documents early on, when no clear hypotheses had been formed. Teams began to clean up the artifacts after successfully finishing or abandoning a line of investigation. After the cleanup, the team would either choose a new analysis path and begin to spread out new documents, or organize the remaining documents to form further hypotheses or connections. Four teams did not clearly exhibit this behavior but instead cleaned up documents right after reading, tracking their progress entirely on paper.

Organization in the Periphery: Reading was very important to the task and document readers could take up half the screen and easily overlap a partner's side. All pairs negotiated a left-right orientation to the screen, reading documents mostly on one side, storing them on the other. In previous work [24], the center of the screen was critical for conducting the groups' main collaborative task. In our study, it was far less important: no pair developed a common group storage space at the center. We hypothesize, that the relatively small width of our table impacted this result as well as the fact that our task did not require groups to create a shared product or artifact during collaboration.

Personal storage piles were created in corners or on the edges of the table by seven pairs. They used the edge of the screen as a site for storage of both relevant documents and searches that might be useful. Figure 5 shows an example of 


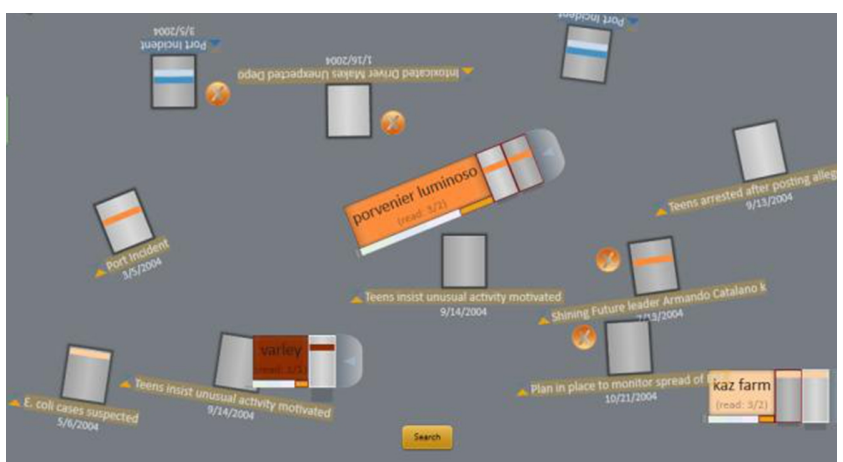

Fig. 4. Typical early organization strategy-documents are spread randomly across the table based on where they were pulled out or read.
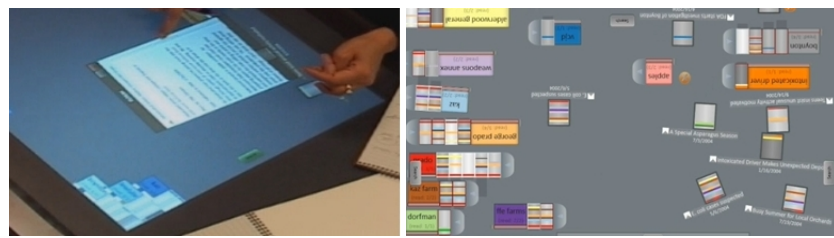

Fig. 5. Organizing important searches and documents in the corners and on the edge.

such an organization. The edges of the screen were typically used for storage after parts of the task had been solved and visual reminders and archives were needed. Teams saw searches as storage containers that kept all the documents relevant to a particular search term. As such, some pairs arranged searches in a vertical column along one side. $23 \%$ of all searches were kept open, rather than minimized to conserve screen real-estate. By leaving search boxes open, relevant and previously accessed documents could be easily found and re-used. In addition, the search boxes acted as a persistent list of keywords that could help the pair track their investigations and connect current insights to past findings. Twelve pairs used their searches this way, and three used them as a shared resource for group access.

Semantic Arrangements: In addition to the loose piles described above, other pairs used the physical space to lay out specific, semantically-inspired placements. Four teams arranged documents on a timeline (Figure 6). Three pairs placed documents based on importance to the overall task, three based on relevance to a specific search theme, and one based on intended next actions (e. g., "to read"). Semantic storage strategies were developed jointly, later in the task, in order to help draw out relationships between documents and facts and to support sensemaking.

In summary, by tracking how pairs organized their documents, this analysis provides insights into how teams thought of the information in the workspace as they made progress on the task. Robinson [23] similarly found-for a different collaborative task - that analysts used several different organization strategies and in different locations of the workspace, further strengthening the finding that workspace organization is important to how people think
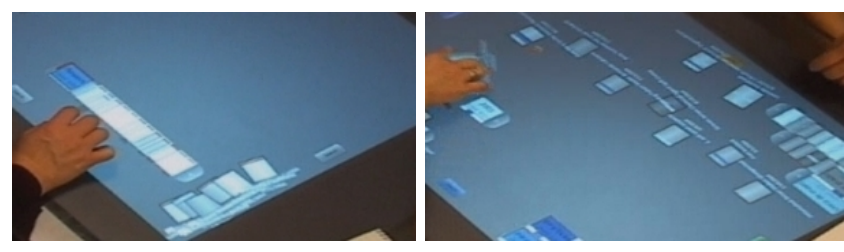

Fig. 6. Organizing documents by importance (left) or by time (right).

of the information they have. We also noted that the organization strategies changed depending on the progress that groups had made. Cambiera made these changes possible by allowing teams to freely organize information. Since the context of a complex task, such as the one we tested, is often dynamic and depends on emerging information, the availability of different organization styles or tools for the team, as well as for the individual team members, is an important consideration for future tools.

\section{Off the Tabletop Workspace}

In order to better understand the role of collaborative work around the tabletop workspace and the interactive capabilities of our software, we also analyzed activities participants engaged in off the tabletop. This analysis provided us with further understanding of the role of our software and the co-located setting in supporting the task.

\subsection{Notetaking}

While Cambiera supports the early phases of the sensemaking cycle, its support for the latter parts is limited. We wanted to further understand how this lack of support for later phases was perceived and handled by participants. We analyzed their comments from the post-session interview and the notes they took during the study.

Four pairs of participants asked for methods to tag documents, and two wanted ways to extract relevant excerpts. These requests point to the need for end-to-end sensemaking support within Cambiera. We followed up these questions by examining the notebooks used by the participants, expecting that many participants would use their notebooks as a place to organize their thoughts. All 15 pairs had at least one person take notes; in total, 24 of the 30 participants took notes. We coded their notepads to understand how they organized information off of the tabletop. 58\% of the people who took paper notes organized at least some of the facts into a timeline. Half of the participants connected names and events with grouping mechanisms, such as circles, brackets, or other annotations. 38\% drew network diagrams to illustrate connections between people and facts. In addition, $25 \%$ of note-takers used the notebook as a to-do list of searches and ideas to follow up. Interestingly, only one pair ever passed their notepads between each other. While it is not clear whether participants simply did not find the need to look at each other's analysis summaries or did not want to share them, our observations suggest that participants 
could have benefited from tools that would suggest overlap between facts that both partners had found. Explicit support of collaborative sensemaking and collaborative reasoning is worthwhile to consider for problem solving tools. Such sensemaking support should offer several means of viewing data, including the timeline, grouping, network, and list views that our participants created in their paper notes.

\subsection{Verbal Communication}

Finally, we analyzed three pairs of participants in detail to better understand the types of information they exchanged verbally and the reasons for these verbal exchanges. This in-depth analysis was conducted for just three of our 15 groups as these types of analyses are extremely timeconsuming requiring the transcription of all verbal communication and several coding passes of these transcripts with accompanying analysis of the $\log$ files. We chose three closely coupled groups $(69 \%, 90 \%$, and $92 \%)$ with high debriefing scores (9-10) and different types of group setups: a group of mixed-gender fellow graduate students, husband and wife, and a pair of same-gender friends and co-workers. We chose three successful groups to be able to observe frequent verbal exchanges and to understand how these may have been beneficial to solving the task. Further analysis could compare our results to those of less successful groups to understand how difference in communication and collaboration led to lower success rate.

\subsubsection{Communication Occurrences}

We based our coding of communication occurrences loosely on the Pirolli and Card sensemaking model [21]. Specifically, we observed communication occur in the following contexts:

Search and Filter: Activities as part of "search and filter" consisted of participants opening the search widget of Cambiera, typing keywords and issuing searches, and looking through the search results to pull out interesting documents. Participants communicated to point out relevant (shoebox) documents to each other, to discuss the connection between documents, to share strategies for searching and reading documents, and to share the number of search results returned by their searches. These verbal communications helped collaborators understand each other's search process and also helped them understand the relevance of documents to the task.

Read and Extract: Participants read documents identified as relevant to the plot and extracted relevant pieces of information and low-level inferences. The evidence extracted was often used to trigger new hypotheses or searches. Of all types of communication "read and extract" was the most common. Participants communicated to point out relevant items and evidence. They also read documents aloud to each other and shared inferences or hypotheses they were forming when reading the documents.

Schematize: Communication of this type occurred when participants began to arrange evidence into timelines, diagrams, tables, and other schemas to build or test hypotheses.
Most schemas were created on paper notepads, though some participants created schemas on the tabletop itself by arranging documents into timelines or piles. Schematizing was often collaborative in that pair members suggested to each other how and when to create schemas. Also, during the creation of schemas, participants often shared the relationship between schema items and read aloud the schema to each other asking whether it seemed accurate based on their current understanding of the problem.

Build Case: The "building case" context consisted of creating and testing hypotheses. Participants verbally shared their individual hypotheses and shared information about evidence. They also discussed what was not known.

Search for Support, Evidence, Information, and Relations: Pairs frequently searched for support or evidence to test their hypotheses. They also searched for relationships between entities and documents. During these phases participants discussed the connections between evidence and previously set aside relevant items. Communication around "search for information" [21] was very prominent. Participants searched for more information about relevant items and then often shared search strategies and the number and quality of their search results, along with sharing the information they already knew about those items.

Sharing Task and Search Strategies: Communication on the best task and search strategies played an important role for our groups but did not map directly to the Pirolli and Card model [21] we used to categorize the other types of communication. Similar to Isenberg et al. [17], we found that pairs often shared task strategies. This included sharing ways for approaching, dividing, and sharing the task, or arranging and organizing documents: e.g., how to read documents together and how to arrange documents in piles or timelines. Along with overall task strategy, participants often discussed search strategies including search keywords to use, the number and types of search results returned, and the shoebox and evidence items they would search for next. These suggestions helped pairs understand how they were each approaching the search process.

\subsubsection{Communication Value}

Communication was an important part of participants' collaboration. While communication was more common in closely coupled styles and during style transition, communication occurred throughout the task. We base our discussion on the value of this communication around the notion of information foraging and sensemaking [21].

Communication occurred while exploring the data space, narrowing down the set of items that had been collected for analysis and examining these documents (information foraging). This helped the pairs to focus their search activities and also cover more data. We found that verbal communications in context to the data on the tabletop display was also important in finding and prioritizing relevant information. This prioritization has been previously discussed as an important component of collaborative sensemaking in the context of collaborative information seeking [25]. During "search and filter", group members 
communicated to point out documents that were important to the task, hence enriching their set of shoebox items; during "read and extract," group members pointed out important phrases and events from the shoebox to each other to enrich their joint set of evidence file items. When individual pair members got "stuck," communicating with their partner helped them move forward with the task. Conducting follow-up searches is a costly activity during sensemaking [21]. We saw group members use each other (instead of documents) as sources of information, thus lowering the cost of follow-up searches. For this reason, asking and answering questions was an important aspect of communication, especially when searching for additional information about shoebox or evidence file items. This may explain why a large number of questions were asked in the "search for information" phase.

In the sensemaking loop [21], sensemakers are focused on generating and evaluating hypotheses, finding evidence to confirm or discard hypotheses, and making decisions. Here, the challenges are to remember and test competing hypotheses and generate alternative hypotheses [21]. We found that during these activities verbal communications were geared towards discussing the relationships between the information that was prioritized during information foraging. Thus, the discussion was focused on connecting evidence, designing schemas, and generating and confirming/discarding hypotheses.

In summary communication between team members was valuable for several reasons:

- to focus search activities \& and cover more data

- to find \& prioritize relevant information

- to transfer shoebox to evidence file items

- to ask for or give help when no progress occurred

- to reduce the cost of follow-up searches

- to connect evidence and design schemas

- to generate and confirm/discard hypotheses

In co-located collaborative work communication is easily engaged in and natural and hence can be extremely beneficial for data analysis. Yet, some of our pairs who worked less closely coupled did not communicate as closely as the three pairs we analyzed in detail. In the next section we discuss how this may have impacted their success on the task and the value of their collaboration overall.

\section{The Value of Collaboration}

We observed a substantial degree of variance in how much team members chose to work together. Groups spent anywhere from $32 \%$ to $92 \%$ of their time in close collaboration. Table 1 gives an overview of the differences in collaboration style across groups. This variability manifested in different amounts of verbal and digital information sharing between groups, impacted how Cambiera's features were used, and also led groups to be more or less successful the task overall. In order to illustrate how different collaboration strategies impacted how well groups performed in the task and how they used Cambiera, we begin by discussing two different teams' strategies in detail.

\subsection{Strategy Examples}

\section{Group 2: Working Closely Together}

This group consisted of two close friends and co-workers. They found all ten critical documents, made all eleven connections with no assists, and solved the complete task correctly in 70 minutes. Both team members worked closely coupled for $90 \%$ of the time (see Table 1). They had a clear work strategy: they searched and browsed the results in parallel but read interesting articles together, and exchanged 13 documents. They were able to rapidly identify connections between facts, and moved through the study very efficiently. Since these participants worked very closely, with frequent communication throughout, they generally had very good awareness of what each other were searching for. Each made sure that their partner read important documents that they had found, so Cambiera's awareness features were less critical for this group. As a result, the pair used query coloring less to track their own searches, and more as a way of finding documents that looked to be informationrich. In particular, they preferentially read documents that had several stripes (found by several searches), and that had not been read before.

\section{Group 5: Failing to Combine Knowledge:}

Group 5 consisted of two friends, co-workers, and experienced puzzle solvers. They had coordinated multi-hundredperson puzzle-solving competitions and felt very confident about their ability to solve the mystery. During the 69 minutes of their work, they found all critical documents but only found five connections and required assistance three times, which was among the worst performance outcome of the study. Both participants reported that they were accustomed to working separately, trying to figure out a puzzle on their own. As a result, they adopted a loosely coupled work style, working closely coupled only $33 \%$ of the time (see Table 1). After reading the initial document together, they each chose a part of the problem that was of interest to them. As they worked, they would look for documents that might help their current approach, and periodically mentioned their thoughts aloud. They spent substantial portions of the task unaware of each others' work. It was not until the experimenter asked for a status update that they began to realize what information they were missing. The group spent much of the study working separately on different problems (DP), periodically checking in. During the task, both partners issued 78 searches; they later reported that they had gotten lost in the sheer quantity of results they had found. Because they spent most of the time working on different problems, they infrequently found overlapping documents; as a result, they almost never used Cambiera's awareness tools.

\subsection{The Value of Close Collaboration}

After closely studying the behavior of our groups we were interested to find out whether the overall pattern of collaboration was linked to how successfully groups were able to solve the task. We calculated a debrief score as 


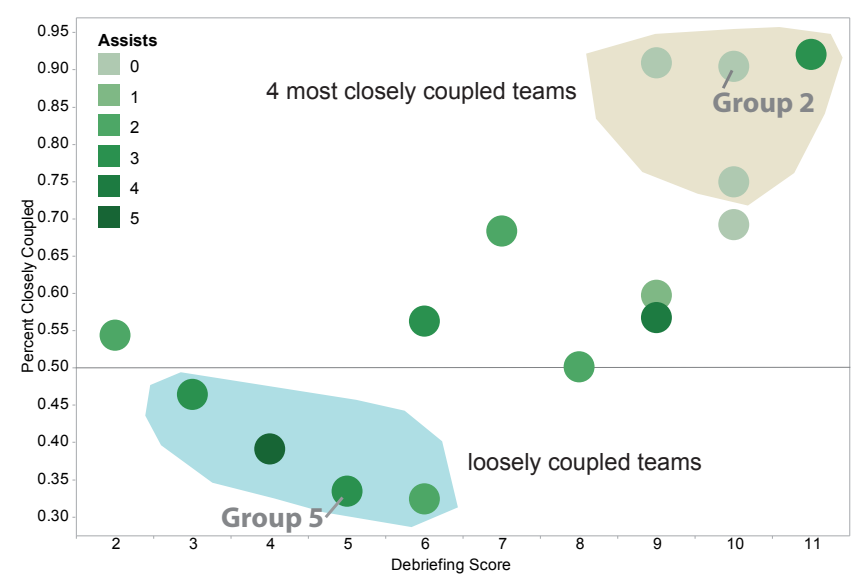

Fig. 7. Relation between debriefing score, time spent closely coupled, and assists per group.

TABLE 2

Debriefing score and assists for the four loosely (bottom) and most closely coupled teams (top).

\begin{tabular}{rrrl} 
Group & Debriefing Score & Assists & Closely Coupled \\
\hline 1 & 11 & 3 & $92 \%$ \\
15 & 9 & 0 & $90 \%$ \\
2 & 10 & 0 & $90 \%$ \\
12 & 10 & 0 & $74 \%$ \\
\hline 3 & 3 & 3 & $46 \%$ \\
8 & 4 & 5 & $39 \%$ \\
5 & 5 & 3 & $33 \%$ \\
7 & 6 & 2 & $32 \%$ \\
\hline
\end{tabular}

proposed for judging the 2007 VAST contest [26] from the debriefing of our participants. At the end of the study we asked participants to report the current hypotheses they had derived. We recorded correctly reported, incorrectly added, and missing facts to form our score. Figure 7 gives an overview of the percentage of time each group spent closely coupled (vertical axis) and their debriefing score (horizontal axis). Except for two outliers, groups that spent more time in close collaboration generally had a higher debriefing score in our study.

Our study differed from the scoring of the VAST contest, however, in that some groups received assists during the study. Whether a group required an assist is another indication of how well it was able to work on the task. The four groups which required no assists spent over 69\% of their time in close collaboration. Loosely coupled teams required between $2-5$ assists. The results for our four most closely and loosely coupled teams are presented in Table 2 and highlighted in Figure 7.

These results suggest that coupling style was linked to teams' success in the task. Pairs that synchronized frequently on their discoveries, in general, did better than those that did not. They connected more facts and required fewer assists. In the task, many of the connections between documents and ideas were subtle and required a leap of insight. For example, participants had to realize that what seemed to be e.coli symptoms in one document might well be a poisoning in another document. Pairs who worked on the same problem ("let's see if we can find other discussions of the poison") were better able to make these connections, while pairs who worked completely independently ("I'll work on the corruption story; you work on the murder") appeared to be less successful. For example, Group 5 (discussed in the previous section) is one example of a group that did not work closely together, and did not perform well on the task overall (see Figure 7). The summaries that they gave each other infrequently included the critical facts that they needed to make connections. Similarly, working too separately was a detriment to other pairs.

Overall, teams that were more successful-that is, reported more correct and fewer incorrect facts, and got fewer hints-were ones that spent more time closely coupled and communicated more frequently. Note that this correlation does not suggest causation: that is, it is unclear whether success on the task leads to coupling or coupling leads to success. It is likely to be a combination of both: in many teams, we observed pairs of participants working independently until one of them found something that seemed promising. Frequently, finding a critical document during separate work led pairs to switch to a more closely coupled style to discuss intermediate results, to read the critical document or other related documents together, to interpret the facts found up to that point, or to offer/ask for help. This was echoed during the debriefing session where eight pairs explicitly stated that they tended to work in a form of divide-and-conquer style. They would first go off and do individual work and then come together when they had found a partial solution or hypothesis to report or wanted to get the other person involved for validation.

\subsection{Suitability of the Tabletop as a Collaborative Visual Analytics Workspace}

During the study, the tabletop was the collaborative center of users' engagement in the task. Cambiera was designed to take advantage of the tabletop setting through features that allowed participants to share and organize information freely similar to the possibilities of a physical setting. Two key benefits arose from using a tabletop that would not have been as accessible in either a multiple-display or a shoulder-to-shoulder configuration: artefact-centered information sharing and direct communication. Both sharing and face-to-face work enhanced collaboration: partners were able to point to and manipulate documents and searches that they felt were relevant, and point to documents that their partners could see. During discussion (DISC) periods, team members did not need to turn away from the table in order to communicate but instead discussions stayed focused on the shared information artefacts, while being able to easily pull in and compare related information. In the debriefing session, one team member said: "sometimes [my partner] and I fight about who gets to use the computer so it was nice to share that. One person standing and another sitting that's never a collaborative act, here we're assembling a 
whole thing together." Others stated that working face-toface helped them, "we kept talking the whole time and we assigned things to each other."

Several team members volunteered that they particularly liked the direct-touch nature of the tabletop for sharing electronic documents. In the debriefing session, 11 of the 15 pairs highlighted the ease of sharing documents as one of their favorite and most useful features of Cambiera. Five pairs expressed that they particularly enjoyed working in a face-to-face setting. Last, the tabletop seemed to allow people to think about the task in a different way: "I liked the direct touch: it's more human, less technical." While some of this enthusiasm can be attributed to the novelty of touch input, all teams approached the table without hesitation and felt encouraged to interact with the system.

The face-to-face tabletop setting and the ability for both partners to synchronously search and read was a strong benefit for the complex problem solving context. We observed several instances in which the collaborative setting contributed to participants making progress in the task. Team members shared and reflected on each others' ideas, cross-validated hypotheses by sharing documents and results, and engaged in repeated discussion about current hypotheses and strategies on how to proceed further (c.f. Section 7.2). All pairs talked to each other, gestured toward searches and documents on the tabletop, and generally utilized the shared reference point of having a tabletop in front of them. In the questionnaire, 23 of 24 participants "agreed" or "strongly agreed" that they knew what their partners were working on, suggesting that the tabletop provided a strong context for mutual awareness.

We note that some teams felt that the Microsoft Surface tabletop, in its current form, was a constraint. At 2' $\times 3$ ', and $1024 \times 768$ pixels, team members often felt cramped, wanting a higher-resolution and physically larger display for document reading. The lack of a physical keyboard meant that typing was cumbersome and error-prone. While many of the tabletops on the market suffer from similar difficulties, some projects are beginning to explore largerscale work surfaces [27] and integrated keyboards.

\section{IMPLICATIONS FOR DESIGN}

In summary, our study found that working face-to-face around the table was a successful way for pairs to solve the complex problem. The setting allowed participants to approach the problem quickly and effectively. All participants immediately immersed themselves in the task and made use of the various features Cambiera offered. One of the surprising findings of our study was that task success and time spent working closely together were highly correlated. This was not a challenge of information finding: every team found most or all of the critical documents; the only question was whether they were able to draw the connections between them. Based on our findings, we draw some design implications for improving Cambiera as well as other co-located collaborative problem-solving tools.

\subsection{Design for Transient Behavior}

The complex problem required collaborators to react to emerging information and hypotheses and required the team to frequently re-assess their work and analysis approach. As there was no obvious best way to solve the task, teams often started out with different strategies. The support of a wide variety of work styles and collaboration strategies is a challenge for the design of collaborative tools. It is insufficient to support just one strategy, since different team members employ different strategies, often in parallel, and pairs frequently shift strategies depending on the current stage of the task. Cambiera, for example, offered flexibility through free workspace organization and repositioning of searches and documents. Team members used Cambiera to engage in different analysis activities in parallel: to search, read, and extract information at any given time without influencing others' work. By allowing team members to create their own copies of documents and search results as needed, and by not forcing participants to explicitly share resources, these flexible work behaviors were able to emerge. We recommend that collaborative systems continue to allow teams to smoothly choose which collaboration style best fits their task requirements and work styles.

Cambiera, however, did not offer sophisticated storage or sharing mechanisms, but participants did request features for relating data and sharing partially-assembled conclusions. The challenge in offering these mechanisms is to design them in a way that they can easily and fluidly be changed, re-appropriated, or morphed depending on task progress and current questions that the team is trying to answer. The current design required people to organize the workspace by themselves, come up with own categorizations or groupings. A future design should include, at minimum, storage containers but simple categorization of items are likely not enough. In particular in later stages of the task people switched to more semantically meaningful organizations and, thus, storage spaces should be enriched with sensemaking support. Automatic organizations according to semantic relationships like time, maps, or social networks could enhance group work. Our groups wanted to be able to link documents together, to add notes and annotations to the tabletop, and to be able to leave comments. In other words, as they learned more about the dataset, they wanted to leave their tracks and thoughts in the data itself.

In summary, for future tools we suggest to specifically focus on storage mechanisms which a) allow for semantic storage by including free as well as automatic organization which takes the data's dimensions into account (e.g., for timelines, maps, social network graphs); and b) include flexible annotation capabilities which allow analysts to draw links between items, circle groups, and label entities within a storage area, and c) allow for flexible re-organization, search, and sharing. 


\subsection{Encourage Closely Coupled Work and Com- munication}

Pairs were able to take advantage of the unique affordances of the tabletop-the face-to-face interaction and direct manipulation of objects - to organize their thoughts, share ideas, and work through the problem. Pairs that worked for too long in parallel were less successful in connecting the facts they found, and so had trouble solving the challenge. Our analysis of communication patterns suggests that it may be particularly important to support communication during the early information foraging phases of the sensemaking process. This could be achieved by providing additional overview visualizations of search results, shoebox items, and evidence file items extracted by all group members. Second, it is important to support focused communication during the sensemaking process. For instance, analysts may want to share priorities assigned to the data, have mechanisms for asking and answering questions related to the data, and explicitly share task and search strategies. Yet, any of these additions have to be designed to allow for fluid interaction with the data and at-a-glance inspection to make the collaboration effortless and reduce the coordination cost between group members.

The awareness features built into Cambiera were meant as an effortless awareness cue to bring teams closer together by encouraging conversation about documents that both team members had read or found. While participants found this subtle awareness information useful and valuable, we conclude that stronger indicators would be more beneficial. These could make more obvious visual connections between common information that team members are reading, that are placed in close proximity, or that have been stored in the workspace. Collaborative annotations and notepads may also have encouraged the loosely coupled teams to re-connect.

\section{Conclusions}

We presented a detailed exploratory study of a complex collaborative problem solving activity around a digital tabletop display. We studied Cambiera, a tool for collaborative analysis work with text document collections. The contributions of this study were a set of findings on our digital tabletop setting as a context for co-located problem solving. We explored its suitability, identified eight collaboration styles which pairs adopted while solving the problem collaboratively, and described how collaboration and communication impacted their success in the task. In particular, our study showed that Cambiera-in the digital tabletop setting-was a successful work context for complex problem solving. Our task required teams to constantly react to new information, to re-interpret what they had found, and to re-assess their strategies. Hence, participants worked together in a variety of work styles, supported by Cambiera's flexible collaborative search, organization, and sharing mechanisms. We found that teams that connected most often about their individual findings, and worked closely together and communicated throughout, were more successful at the task and required fewest assists. Based on these observations, we offer recommendations for features that could be used to improve co-located problem-solving tools more generally. In particular, support for teams to make ad-hoc changes to all aspects of their current work strategies as well as features that encourage them to share information and connect with each other frequently, are worth considering.

\section{ACKNOWLEDGMENTS}

We thank our participants for taking part in the study, our co-workers at Microsoft for their help with pilot studies, and our reviewers for valuable feedback.

\section{REFERENCES}

[1] B. Mirel, Interaction Design for Complex Problem Solving. San Francisco: Morgan Kaufmann, 2004.

[2] G. Chin, Jr., O. A. Kuchar, and K. E. Wolf, "Exploring the analytical processes of intelligence analysts," in Proc. of CHI. New York, USA: ACM, 2009, pp. 11-20.

[3] P. Isenberg and D. Fisher, "Collaborative Brushing and Linking for Co-located Visual Analytics of Document Collections," Computer Graphics Forum, vol. 28, no. 3, pp. 1031-1038, Juni 2009.

[4] P. Isenberg, D. Fisher, M. Ringel Morris, K. Inkpen, and M. Czerwinski, "An Exploratory Study of Co-located Collaborative Visual Analytics around a Tabletop Display," in Proc. of VAST. Los Alamitos, USA: IEEE, 2010, pp. 179-186.

[5] A. D. Balakrishnan, S. R. Fussell, and S. Kiesler, "Do visualizations improve synchronous remote collaboration?" in Proc. of CHI. New York, USA: ACM, 2008, pp. 1227-1236.

[6] A. D. Balakrishnan, S. R. Fussell, S. Kiesler, and A. Kittur, "Pitfalls of information access with visualizations in remote collaborative analysis," in Proc. of CSCW. New York, USA: ACM, 2010, pp. $411-420$.

[7] S. E. Brennan, K. Mueller, G. Zelinsky, I. Ramakrishnan, D. S. Warren, and A. Kaufman, "Towards a Multi-Analyst, Collaborative Framework for Visual Analytics," in Proc. of VAST. Los Alamitos, USA: IEEE Comp. Society, 2006, pp. 129-136.

[8] S. A. Paul and M. R. Morris, "Cosense: Enhancing sensemaking for collaborative web search," in Proc. of CHI. New York, USA: ACM, 2009, pp. 1771-1780.

[9] P. E. Keel, "Collaborative Visual Analytics: Inferring from the Spatial Organization and Collaborative Use of Information," in Proc. of VAST. Los Alamitos, USA: IEEE, 2006, pp. 137-144.

[10] F. B. Viégas, M. Wattenberg, F. van Ham, J. Kriss, and M. McKeon, "Many Eyes: A Site for Visualization at Internet Scale," IEEE Transactions on Visualization and Computer Graphics, vol. 12, no. 5, pp. 1121-1128, Nov./Dec. 2007.

[11] P. Isenberg, M. Sedlmair, D. Baur, T. Isenberg, and A. Butz, "Proceedings of the VisWeek workshop on Collaborative Visualization on Interactive Surfaces-CoVIS 2009," Ludwig Maximilias University Munich, Munich, Technical Report LMU-MI-2010-2, April 2010.

[12] M. R. Morris, J. Lombardo, and D. Wigdor, "WeSearch: Supporting Collaborative Search and Sensemaking on a Tabletop Display," in Proc. of CSCW. New York, USA: ACM, 2010, pp. 401-410.

[13] C. Forlines and C. Shen, "DTLens: Multi-user Tabletop Spatial Data Exploration," in Proc. of UIST. New York, USA: ACM, 2005, pp. 119-122.

[14] P. Isenberg and S. Carpendale, "Interactive Tree Comparison for Colocated Collaborative Information Visualization," IEEE Transactions on Visualization and Computer Graphics, vol. 13, no. 6, pp. 1232 1239, Nov./Dec. 2007.

[15] D. Wigdor, H. Jiang, C. Forlines, M. Borkin, and C. Shen, "WeSpace: The Design Development and Deployment of a Walk-up and Share Multi-Surface Visual Collaboration System," in Proc. of CHI. New York, USA: ACM, 2009, pp. 1237-1246.

[16] J. Stasko, C. Görg, and Z. Liu, "Jigsaw: Supporting Investigative Analysis Through Interactive Visualization," Information Visualization, vol. 7, pp. 118-132, 2008. 
[17] P. Isenberg, A. Tang, and S. Carpendale, "An Exploratory Study of Visual Information Analysis," in Proc. of CHI. New York, NY, USA: ACM, 2008, pp. 1217-1226.

[18] A. Tang, M. Tory, B. Po, P. Neumann, and S. Carpendale, "Collaborative Coupling over Tabletop Displays," in Proc. of CHI. New York: ACM, 2006, pp. 1181-1290.

[19] G. Grinstein, T. O'Connell, S. Laskowski, C. Plaisant, J. Scholtz, and M. Whiting, "VAST 2006 Contest-A Tale of Alderwood," in Proc. of VAST. Los Alamitos, USA: IEEE, 2006, pp. 215-216.

[20] C. Andrews, A. Endert, and C. North, "Space to Think: Large HighResolution Displays for Sensemaking," in Proc. of CHI. New York, USA: ACM, 2010, pp. 55-64.

[21] P. Pirolli and S. K. Card, "The sensemaking process and leverage points for analyst technology as identified through cognitive task analysis," in Proc. of the Conference on Intelligence Analysis, 2005.

[22] P. Isenberg and D. Fisher, "Pairgrams: Understanding Collaborative Analysis Behavior with Visualization," in CHI Workshop on Analytic Provenance: Process+Interaction+Insight, Held at the Conference on Human Factors in Computing Systems (CHI), 2011.

[23] A. Robinson, "Collaborative synthesis of visual analytic results," in Proc. of VAST. Los Alamitos, CA, USA: IEEE, 2008, pp. 67-74.

[24] S. D. Scott, M. S. T. Carpendale, and K. M. Inkpen, "Territoriality in Collaborative Tabletop Workspaces," in Proc. of CSCW. New York, USA: ACM, 2004, pp. 294-303.

[25] S. A. Paul and M. Reddy, "Understanding together: Sensemaking in collaborative information seeking," in Proc. of CSCW. ACM, 2010, pp. 321-330.

[26] C. Plaisant, G. Grinstein, J. Scholtz, M. Whiting, T. O'Connell, S. Laskowski, L. Chien, A. Tat, W. Wright, C. Görg, Z. Liu, N. Parekh, K. Singhal, and J. Stasko, "Evaluating Visual Analytics at the 2007 VAST Symposium Contest," IEEE Computer Graphics and Applications, vol. 28, pp. 12-21, 2008.

[27] B. Hartmann, M. R. Morris, H. Benko, and A. D. Wilson, "Augmenting Interactive Tables with Mice \& Keyboards," in Proc. of UIST. New York, USA: ACM, 2009, pp. 149-152.

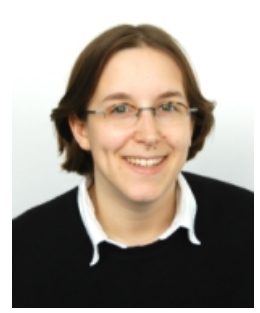

Petra Isenberg is a full-time research scientist at INRIA, Saclay, France. She holds a PhD from the University of Calgary and a Diplom-degree in Computational Visualistics from the University of Magdeburg. Her research interests include information visualization, visual analytics, large interactive surfaces, and computer-supported cooperative work. Her particular research focus is collaborative visualization and visual analytics.

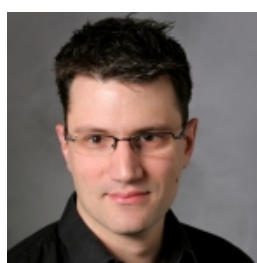

Danyel Fisher is a researcher at Microsoft Research's VIBE (Visualization and Interaction) team. His research interests center on information visualization and online collaboration, and the ways that visualizations can be used together. Danyel received his PhD from the University of California, Irvine, in 2004.

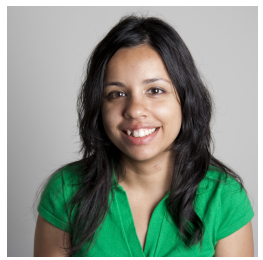

Sharoda A. Paul is a Computing Innovation Fellow in the Augmented Social Cognition group at the Palo Alto Research Center (PARC). She holds a Ph.D. in Information Sciences and Technology from Penn State University. Her research interests are in computer-supported cooperative work, human-computer interaction, collaborative and social Web search, social computing, and healthcare informatics.

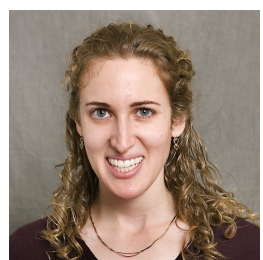

Meredith Ringel Morris is a research scientist in the Adaptive Systems \& Interaction Group at Microsoft Research. She is also an affiliate assistant professor at the University of Washington. Dr. Morris's research area is human-computer interaction, with an emphasis on computer-supported cooperative work and social computing. She was named one of 2008's 35 Innovators Under 35 by Technology Review, and one of 2009's 100 Notable Women in Seattle Technology by TechFlash.

Dr. Morris earned a Ph.D. \& M.S. in computer science from Stanford University, and an Sc.B. in computer science from Brown University.

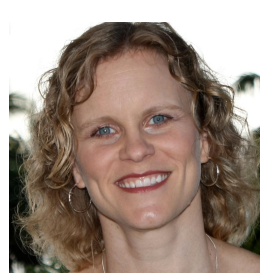

Kori Inkpen is a researcher in the Visualization and Interaction Group (VIBE) at Microsoft Research and manager of the CONNECT group. Prior to joining Microsoft Research Kori was a Professor of Computer Science at Dalhousie and Simon Fraser University. She holds a PhD from the University of British Columbia. Her main research area is Human Computer Interaction with a focus on computer-supported collaboration. Kori is interested in exploring ways to support collaboration across a variety of domains including home, work, education, healthcare, and fun.

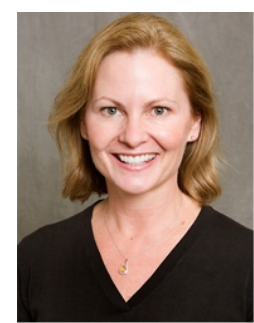

Mary Czerwinski is a Research Area Manager of the Visualization and Interaction (VIBE) research group at Microsoft. She holds a Ph. D. in Cognitive Psychology from Indiana University in Bloomington. Mary has been an affiliate member of the Psychology Departments at the University of Washington and Rice University and sits on several academic and professional advisory boards. Much of Mary's work focuses on novel information visualization and interaction techniques. She is a distinguished scientist of the ACM and was recently elected into the association's $\mathrm{CHI}$ Academy. 